\title{
Organisational Learning with SaaS CRM - A case study of Higher Education
}

\section{Taiwo Oseni}

Centre for Informatics and Applied Optimization

Federation University Australia

Ballarat, Australia

Email: t.oseni@federation.edu.au

\section{Mehmood Chadhar}

Centre for Informatics and Applied Optimization

Federation University Australia

Ballarat, Australia

Email: $\underline{\text { m.chadhar@federation.edu.au }}$

\section{Sasha Ivkovic}

Centre for Informatics and Applied Optimization

Federation University Australia

Ballarat, Australia

Email: s.ivkovic@federation.edu.au

\section{Sally Firmin}

Centre for Informatics and Applied Optimization

Federation University Australia

Ballarat, Australia

Email: s.firmin@federation.edu.au

\section{Abstract}

Customer Relationship Management (CRM) generally has a reputation as a technology that does not live up to its over-inflated expectations. Yet, implementations in higher education remain on the rise. Higher Education institutions (HEIs) are embracing cloud-based CRM systems to upsurge performance, encourage better management practices, and enhance their relationship with staff and students. CRM success however relies heavily on an adaptive organisational learning (OL) process upon which proactive decisions can be made. This paper emphasises that committed learning in post-implementation use is paramount to attaining further understanding of the capabilities, features and functionality of the CRM. Investigating how SaaS CRM usage reflect an organisation's learning in a Higher Education context, the paper presents theoretical and practical contributions in a framework for effective SaaS CRM utilisation, and recommends a continuous cycle of exploration-exploitation-exploration. Yet the reality is that organisations explore, exploit, and then stop exploring.

\section{Keywords}

CRM, SaaS, Organisation Learning, Exploitation - Exploration, Higher Education 


\subsection{Introduction}

Organisational learning (OL) is key to organisations' efficient and effective use of new technology. Taking different forms, OL leads to the creation of various types of organisational capabilities, some leading to improvements of existing organisational routines, and others culminates in the development of entirely new routines (Linda \& Manpreet, 2017). Not surprisingly, the 2017 Gartner's Hype Cycle reports that cloud-based Information Systems (IS) are now in a phase where focused experimentation and solid hard work by organisations lead to a true understanding of the technology's applicability, risks and benefits (Smith \& Anderson, 2017). With digital business increasingly becoming crucial to remaining competitive, the SaaS (Software as a Service) model of cloud-based systems is more and more being adopted by organisations. Offering greater flexibility and agility that enable organisations to concentrate on their core businesses and competencies (Bernsteiner, Kilian, \& Ebersberger, 2016). The SaaS market is expanding and gradually having significant impact on different industry sectors, including Higher Education sector. Higher Education institutions (HEIs) are especially beginning to embrace cloud-based Customer Relationship Management (CRM) systems to upsurge performance, encourage better management practices, and enhance their relationship with staff as well as existing and prospective students (Amuna, Shobaki, Naser, \& Badwan, 2017). The SaaS model is particularly attractive, being the easiest cloud-based offering since all services are provided and bundled in the delivered software (Buxton, 2015). Faced with technological and economic changes, HEIs such as universities are under increased pressure to enhance value and effectiveness as far as current and potential customers are concerned, and as such find 'easily deployed' SaaS CRM desirable. In HEIs, the concept of students and faculty staff members as "customers" has become a competitive imperative with a profound impact on how colleges and universities attract, retain, and serve customers of all types (Futa, 2004). An educational CRM system aids HEIs to have a full understanding of students' requirements as it collects customer knowledge in all phases of student interaction phases: admission, fee payment, registration, course conclusion, etc. (Abubakar \& Mukhtar, 2015). Expectedly, CRM implementations in higher education hit its peak in 2016; yet because CRM generally has a reputation as a technology that does not live up to its over-inflated expectations (Williams, 2017), it remains an important area to be researched. We suggest in this paper that for investments in CRM live up to expectations, organisations must consider their approach to learning. Our recommendation is aligned with previous research findings about the contribution of organisational learning in organisations' justifications for investment in similar enterprise systems (Chadhar \& Farhad, 2017; Janson, CecezKecmanovic, \& Zupančič (2007). According to Peltier, Zahay, and Lehmann (2013), CRM success relies heavily on having an adaptive organisational learning process upon which proactive decisions can be made. A learning organisation draws on and integrates strategic and tactical insight across functional areas in an organisation and such learning spans vertical and horizontal organisational units, including organisational culture and cross-functional/intradepartmental learning processes(Chadhar \& Farhad, 2017; Janson, Cecez-Kecmanovic, \& Zupančič (2007). Organisations must be invested in educating users, discovering, understanding, learning to use, and actually using the capabilities and features included in CRM (Seethamraju, 2015). This draws attention to the relevance of organisational factors such as learning in CRM use as without committed investment in post-implementation use, it becomes difficult to attain further understanding of the capabilities, features and functionality of the CRM.

The purpose of this study is therefore to consider the organisational elements, particularly organisational learning, and its relevance in successful post-implementation use of a SaaS CRM System, with a Higher Education institution as the context of investigation. This objective will be achieved by addressing the question: How does SaaS CRM usage reflect an organisation's learning?

The paper presents theoretical and practical contributions in a framework for effective SaaS CRM utilisation, and recommends that a continuous cycle of exploration-exploitation-exploration is necessary.

The rest of the paper is structured to include a review of the SaaS CRM and organisational learning literature in Section 2, followed by a discussion of the research methodology in Section 3. Sections 4 and 5 presents the case study and discussions respectively. In section 6 , the paper is concluded, and further research recommended. 


\subsection{Literature Review}

\subsection{SaaS CRM - why organisational learning matters}

Other cloud-based models, Platform-as-a-Service (PaaS) and Infrastructure-as-a-Service (IaaS), are frequently hailed as fundamental to cloud computing, SaaS remains a model for technology to drive and deliver innovation in businesses (Buxton, 2015). The only infrastructure needed for SaaS being broadband Internet connectivity, SaaS has proved it is here to stay. The multi-tenancy model of SaaS allows organisations to scale rapidly without incurring additional infrastructure or staffing costs, and service providers can readily support customers in responding to changing market requirements faster than with on-premise software. More importantly, SaaS solutions comply with the demand for digital transformation, and can be provisioned with great speed, often with simply a browser and an Internet connection from a wide range of desktop and mobile devices (Buxton, 2015).

As customers are aware that a SaaS model may simplify the introduction of a new software system, they usually have high expectations concerning the usability of the software, and in many cases expect an easy start (Bauer, 2012). Such expectations are logical given that the software is readily available on the Internet and can immediately be used. This is one of the advantages of SaaS solutions, there are implications regarding how organisations approach the organisational change associated with such adoptions. For instance, such expectations may mean that most organisational staff are not willing to invest extensive effort in reading user manuals or conduct new training. As a new technology, the organisation's approach to learning is important and should emphasise the relevance of engaging in cross-departmental discussion and learning activities that help to adapt to the new SaaS CRM system (Chen, Wu, Chu, Lin, \& Chuang, 2017).

Son and Lee (2011) suggest that high organisational learning capacity increases cloud computing adoption, and Malladi and Krishnan (2012) found that a firm's existing strengths in organisational learning, coordination IT capability and process management have a role in enhancing the impact of the technology. These studies highlight organisational learning as an important part of embracing cloud computing services in organisations. For instance, organisational culture, staff skills, and work practices can be expected to change as SaaS solutions are adopted and utilised (El-Gazzar, 2014), pointing to the fact that the manner in which an organisation learns will impact on how effectively the organisation uses the SaaS system. We draw on this in our efforts to understand the role of organisational learning on how a cloud-based system, in this case a SaaS CRM can be utilised to bring about expected benefits.

The introduction of cloud-based enterprise services does alter roles and responsibilities (Bernsteiner et al., 2016). For instance, new roles will emerge, like managers for the different types of services, service architects or staff that are responsible for service orchestration. Such new roles add value to the organisation as they influence the skills of employees who would have to develop an understanding on how these kinds of IS work and what kind of possibilities they offer. Consequently, organisational elements such as structure as well as internal and external processes may be impacted. However, as per authors' knowledge, such impacts of organisational elements, particularly organisational learning, are not investigated and only little work has been done in considering the role of organisational learning in the achievement of expected benefits from such adoptions as SaaS CRM. This research aims to fill this research gap by investigating how SaaS CRM usage reflect an organisation's learning.

\subsection{Organisational Learning Types - Exploitation and Exploration}

Many definitions of organisational learning concern the active use of data in guiding organisational behaviour (Edmondson \& Moingeon, 1998). In specific relation to IS, Tomblin (2010) argues that organisational learning describes the efficient application of captured and assimilated knowledge to achieve positive influences on organisations' IT infrastructure as well as business experience. One prominent theory of organisational learning is the Exploitation-Exploration learning introduced by March (1991). According to March (1995), in order to improve a firm's performance, learning involves a trade-off between exploitation and exploration, where exploitation organisational learning is described as the refinement and extension of existing competencies and exploration organisational learning as discovery and innovation. In other words, the utilisation, refinement and extension of existing capabilities is considered to be exploitation, whereas the search for alternative capabilities that are able to strengthen future exploitative potential is explorative learning (Yamin \& Sinkovics, 2007). Three important characteristics for differentiating between exploitation and exploration learning include the objectives, the type of activities through which learning is accomplished, and the timing of benefits derived (Hunter, 2003). Each characteristic can be identified by indicators. Adapted from (Hunter, 2003), Table 1 summarises the indicators of exploitation and exploration in learning as defined by the theory (March, 1991, 1995; March, Sproull, \& Tamuz, 1991). 
Table 1: Distinctions between exploitation and exploration organisational learning

\begin{tabular}{lll}
\hline Characteristics & Exploitation Indicators & Exploration Indicators \\
\hline Objective & $\begin{array}{l}\text { Increase in reliability } \\
\text { Accuracy and control over core processes } \\
\text { Improvement of short-run efficiency } \\
\text { Refinement of existing } \\
\text { capabilities/technologies }\end{array}$ & $\begin{array}{l}\text { Development of new knowledge } \\
\text { New ways of problem- solving } \\
\text { New strategies and technologies }\end{array}$ \\
& $\begin{array}{ll}\text { Activities that emphasise a reduction of } \\
\text { variation in organisational processes and }\end{array}$ & $\begin{array}{l}\text { Activities leading to an increase in } \\
\text { variation in organisational processes, } \\
\text { tasks and functions. }\end{array}$ \\
& $\begin{array}{l}\text { activities. } \\
\text { Activities are risk-aversive in nature }\end{array}$ & $\begin{array}{l}\text { Activities involve risk-taking, } \\
\text { innovation, research }\end{array}$ \\
& & Uncertain and remote \\
\hline Outcomes & Certain and immediate &
\end{tabular}

Given the inherent tension between exploitation and exploration, the tendency to explore versus exploit is affected by several factors such as resources, organisational culture, and structure and a focus on how one learning type disrupts the other (Lavie, Stettner, \& Tushman, 2010). For instance, organisations that focus on exploitation trade flexibility for stability, while those that focus on exploitation may face challenges when attempting exploration. Specifically for the context of Cloud CRM post-implementation usage, as organisations choose 'use' scenarios that emphasise reduced variation in organisational processes as opposed over ones that lead to an increase in variation, they may be favouring exploitation against exploration. Similarly, when 'use' scenarios involve innovation in business processes, it may be the case that exploration chosen over exploitation.

\subsection{Exploitation and Exploration in Cloud CRM post-live use}

Our argument in this study is that organisational learning will likely inform the manner in which organisations use their Cloud CRM systems, with their use scenarios indicating either exploitation or exploration learning. Exploitation and exploration organisational learning concepts have been previously used for classifying various organisational projects: technological projects (Hunter, 2003), organisational innovations (Li, Vanhaverbeke, \& Schoenmakers, 2008), product innovation (Greve, 2007), on-premise enterprise systems implementation (Kraemmerand, Møller, \& Boer, 2003; Tomblin, 2010), and ERP post-implementation modifications (Oseni, Rahim, Smith, \& Foster, 2012; Oseni, Rahim, Smith, \& Foster, 2014). Organisational learning in terms of exploitation and exploration has however not been adopted as a lens for CRM adoptions. Yet, exploitation and exploration will likely occur as an organisation, through the use of CRM, transforms data to information and information to knowledge. In addition, as the use of traditional in-house and packaged software such as ES, has been found to result in organisational learning (Edberg \& Olfman, 2001a; Edberg \& Olfman, 2001b), which then influences how ES are maintained and enhanced (Kraemmerand et al., 2003; Nicolaou, 2008; Tomblin, 2010). How organisational learning influences the manner in which such packaged software are utilised is however an area that is presently not covered by the literature. Particularly, as onsite implementations are decreasing and cloud based options are more desirable, investigating exploitation and exploration tendencies appear important. This is especially the case because organisational learning significantly impacts on the assimilation of cloud technology (Bharati \& Chaudhury, 2014), alongside influencing organisational elements such as culture, staff skills, and work practices (El-Gazzar, 2014).

Based on the different features that characterise each learning type, we suggest that SaaS CRM use scenarios may indicate organisational learning through exploitation and exploration. Hence our goal in this paper is to investigate how the use of cloud CRM demonstrates exploitation vs exploration, and present strategies for effective SaaS CRM usage. This is relevant and emphasises that attachment to the existing core competencies makes organisational learning and the development of fundamentally new capabilities extremely difficult for established organisations (Khanagha, Volberda, Sidhu, \& Oshri, 2013). The paper therefore considers organisational learning in successful post-live use of SaaS CRM, with a Higher Education institution as the context of investigation and seeks to explicate how SaaS CRM usage reflect an organisation's learning.

\subsection{Research Method and Context}

The research approach for the current study is an interpretive case study. The approach is adopted to gain insight into how organisational learning emerges in SaaS CRM use. The revelatory aims and research question of the current study inclined us towards an exploratory case study. A case study is defined as a research strategy that examines a phenomenon in its natural setting, and permits the researcher to gather information from one or multiple entities by employing multiple data collection 
methods (Benbasat, Goldstein, \& Mead, 1987; Yin, 2009). A single exploratory case study provides detailed and intensive analysis of the phenomenon of interest (SaaS CRM use), with emphasis on an intensive examination of the setting (Bryman, 2012). Our case study will explore the concepts of organisational learning for understanding how SaaS CRM can be better utilised. We present a case study of a higher education institution reflecting SaaS CRM adoption and use from an OL perspective by questioning several use cases as reflecting exploitation or exploration OL. The selection of an Australian higher education institution is purposive and not random. This provides a suitable context for the research questions to be answered (Yin, 2009). As higher education CRMs normally serve three key audiences: prospective students, current students, and alumni/donors (Vugt \& Knasys, 2015), they differ from CRM Systems that are designed for a single type of customer. Data is collected via interviews with three key CRM users: ITS Program Manager who managed the implementation of CRM, Student Systems and Processes Manager who handles the day to day running of the CRM, and the Student Experience Coordinator who coordinates the use of the CRM for business processes. These three key personnel were engaged as they represent the major informants for the CRM implementation and postimplementation use. To identify what mode of learning has been employed in a use scenario, interview questions cover skills, procedures or capabilities that were improved, challenged or changed during that particular use scenario. Questions are designed to identify exploitation activities which can be characterised as needing already accumulated knowledge, clear, able to be properly conducted using present knowledge, focused on achieving short-term goals and which are clearly consistent with existing company policy. For exploration CRM use, questions sought to uncover activities which represent a search for new possibilities within the context of SaaS CRM use, as well as an evaluation of diverse options with respect to business processes, which might require staff members to learn new skills or obtain new knowledge. Data is analysed thematically using a pattern-matching logic (Yin, 2009), a technique of analysis which allows comparisons between empirically-based patterns and conceptual predictions. Following the recommendation of Kelle (2007), qualitative coding was done to develop theoretical concepts and categories from unstructured textual data.

\subsection{Alpha University Case study - Data Analysis}

\subsection{Implementation Background}

This is a case of SaaS CRM implementation and usage at a higher education institution in Australia Alpha University; a 1.5 million dollar government-funded project with the purpose to address problems with student engagement. Prior to the initiation of the project, the university was new to the SaaS environment as most enterprise systems used by the university had been hosted on the premise. The strategy with the cloud CRM was to have a template based implementation with a reliance on "AS IS" functionality. Microsoft dynamics CRM was selected and two of its modules were implemented. The two modules delivered were considered a success from a products' delivery point of view, holistically the system is not used for engaging students through the entire student lifecycle. In addition, though the system offers data mining and analytics functionalities, these are not presently being used. In consideration of its use from an OL perspective, the objectives of the implementation, associated activities, and resulting outcomes are investigated. As presented in Table 1 above, three important characteristics for differentiating between exploitation and exploration learning include the objectives, the type of activities through which learning is accomplished, and the timing of benefits derived. These three aspects are able to indicate Exploitation/Exploration organisation learning, and therefore form a lens through which we discuss findings from the case.

\subsection{Implementation Objectives}

Exploitation can be reflected in objectives for the use of CRM such as for increased reliability, accuracy and control over core business processes, including improvement of short-run efficiency, and refinement of existing capabilities/technologies. Objectives that include the development of new knowledge, new ways of problem- solving, and new strategies and technologies on the other hand reflect exploration learning. From this perspective, the adoption of SaaS CRM can be seen to reflect exploration by the university. The CRM project was an opportunity to invest in a gap that existed. Because there were no systems and sheets for capturing data of student interactions, the system was considered because it had embedded in it, processes that were desired but at the moment not in existence. This can be observed in the statements below:

"Prior to this, a student management application was used but the marketing team was missing a function to engage with prospective students. There was no process of interacting with such students and so there was a big hole in that respect. The student customer services were using their internal sheets and there was no mechanism to obtain data that can be used to see the type, nature, 
and interest of students and reassessing...so that was a key reason for the project" - ITS Program Manager

"We had very fragmented student systems and processes, and nothing that could record interactions with prospective students...no consistency. One of the aims was to have a single repository for students management. I think it was recognised that we needed a better way to deal with students communication. There is value in having a system where communication is recorded and it could be accessed by anyone that needed to look into the records" - Manager, Student Systems and Processes

The project represented a new venture where the university did not have much expertise or knowledge, also pointing to exploration as mentioned below:

\begin{abstract}
"On a strategic level, we did not have a vast knowledge of how well CRM could be used ...We also didn't have a CRM support person to assist us in exploring new possibilities... The system was put in so we could understand what kind of enquiries we get, and to ensure that we had relevant staff available to manage the enquiries...for instance to understand what time of the year we get an influx of enquiries, what piece of work do we need to do, the staff we'll need to be responding to enquiries..."-Student Experience Coordinator
\end{abstract}

\title{
4.3 Implementation Activities
}

Exploitation activities emphasise reduced variation in organisational processes and are risk-aversive in nature while Exploration activities lead to increased variation in organisational processes, tasks and functions, and involve risk-taking, innovation, and research. The objectives of the project point to exploration, the actual use of SaaS CRM in the university point to exploitation. The activities undertaken in using the new system highlight the desire for reduced variation in processes. For instance, the ITS program manager stated:

"When human do things, they actually do it based on whatever they think is right, but when you go for standardisation and best practices...you've got options to customise or use as is..and if you use as is then you change your business processes, you get trained on them and try to see things in a different way from how you were doing things .. and this is the kind of learning that a department or unit goes through with exposure to a new system...but that is not the case here" - ITS Program Manager

Similarly two other users express a similar sentiment reflecting that the use of the system does not reflect a search for possibilities, but rather emphasise well-known organisational processes.

“We're basically doing the same job...we answered emails before, we're answering emails now just using a different system... So instead of the email coming into the different mail boxes, it comes straight into CRM"-Student Experience Coordinator

"Since the initial CRM implementation, there have been plans to do a phase 2 but that that hasn't happened...so at the moment its just business as usual but using the CRM... Up until now its been just using the system for what we know or what we've been told that the system can do... we haven't had time to go back to find out what else we can do. ..we know what we know because we know it, but theres a whole lot more functionality that we can be using that we just haven't investigated "Manager, Student Systems and Processes

\subsection{Implementation Outcomes}

Similar to the activities, the outcomes reflect exploitation through automated processes and reflected in certain and immediate benefits. With exploration, while benefits are expected, there usually will be some uncertainty associated and a lower level of clarity as to what the outcomes might be.

\footnotetext{
"As per outcomes, people are happy because their processes are no longer manual and the system is delivering some key critical functionality that are beneficial, but if you look at it holistically, it's not doing what we put it in for considering all the money we spent ... now we are considering whether to go for another system or to re-implement other modules from dynamics into our current environment... it could have been better and there is obviously need for improvement but there are talks to dump it and bring something new. ”- ITS Program Manager
}

"It previously took about 5 days to respond to enquiries, now that's reduced to 2 days...the right people get allocated the job not like before where mails got allocated to people who really could not respond appropriately... and we have a single view of student interactions its easier for us now to create folders for each staff member to ...students are going to get correct response all the time...Its easier to allocate work to the team"--Student Experience Coordinator 
"All emails come in are now directed to CRM and that's become a single point for monitoring and responding to emails. We previously couldn't quantify that sort of traffic. Managers are using the system to manage workload such that if it's a busy period, more people are directed to look at emails" - Manager, Student Systems and Processes

\begin{abstract}
"When you bring in a new system, it brings new functionality that impacts on business processes so there is automation definitely on the business front ...many things have become system driven and one of the major functionalities used is the prospects where the system captures the information and introduces a few perks ..prior to this, all that was done was to gather information from emails in a manual way then the system captures and analyses the information...but I believe they could have really changed the way that has been done in the past and this was the main trigger point for the project”- ITS Program Manager
\end{abstract}

\title{
5.0 Discussion
}

Following data collection and analysis, we now present our discussion in two levels: first the findings of our study, and subsequently our reflection of the findings.

\subsection{Findings}

Assessing the objectives, activities, and outcomes of the SaaS CRM project through discussions with major stakeholders at the university highlight that while exploration was desired at the initiation of the system, in reality, Exploitation was undertaken. The project's objectives reflect exploration while the project activities and outcomes reflect exploitation. This instigates an important question: what happened during the implementation journey to redirect Exploration into Exploitation? As indicated by the ITS Programs Manager, the delivery of two modules was considered successful because in the end, there was a new product. In a more rounded perspective however, it seemed that only a tiny portion of available functionality is being exploited. For instance, the system can be used for engaging students from prospects to the entire student lifecycle of but isn't been used as such. Yet, the university is considering abandoning the system and re-implementing a new system.

So the question, why do organisations explore, exploit, and then stop exploring? Why do they prefer to explore a new system that engage in further exploration of the old? There is obviously more that can be done. For instance, data mining and analytics can be explored but isn't. This was indicated by the IT Program manager, Student experience coordinator, and manager of student systems.

\begin{abstract}
"Analytics and business intelligence are other key things that are important...while the system may have data mining and analytics, we are not using it...we are at the moment not performing any data analytics functionality from the CRM system...there is a lot more opportunity...Half of the battle is won by delivering critical functionality...but more importantly, further exploration, identification and implementation is required because that is going to bring the entire cycle to a closure and will guarantee an ongoing live relationship between a university and its customers" ITS Program Manager
\end{abstract}

"There is more and we really need to start looking beyond what we are currently using them for. We need to go in and gave a play and see how things work... an outside of the box functionality we can use, and there'll be things that will be bigger and we may consider as projects. We need to be asking questions. If the system doesn't work the way we thought it'll work, does it meet our needs or there's this add on that we might be able to buy that will deliver more?" - Manager, Student Systems and Processes

In summary, it does appear that effective use of the system will be a combination of exploitation and exploration. Exploitation seems to come easily and prompts a consideration of hindrances to exploration? A few things can be attributed from this case.

- First, it does appear that a lack of sufficient knowledge discourages exploration. The student experience coordinator for instance expressed that the much that could be done using the system required specialty knowledge that the team did not have at the time:

"We were also looking to be able to perform some reports using the system but that has not happened yet because the reports are really not good... If we can get the reports up and running that will be good...we will be able to look through the entire student lifecycle and we know where staff are needed...Are the moment, the reports are crap...generic and not customised for our need...the reports have not been designed to suit us..we got the package but we did not have the support we needed"-Student Experience Coordinator

The absence or externalising of such relevant knowledge has implications for effective use of the CRM. For instance, without adequate knowledge, an organisation cannot strengthen its 
capabilities in the area. Internalising such knowledge presents organisations the opportunity of learning-by-doing, thereby strengthening its capabilities (Linda \& Manpreet, 2017).

- Second, Exploration may require additional resources. The student services manager expressed this by indicating that an additional software will be required should they decide to pursue the idea of expanding the functionality provided by the CRM to meet specific needs:

"There is a lot more potential and we are starting to look at how we can use the CRM for capturing and engaging with social media. At the moment, we are using CRM to send emails to students but we're also really interested in how we can use CRM to contact students via SMS. That obviously involves some other software rather than expecting students to read emails because they really don't...Timetabling for example is looking at being able to let students know when classes are cancelled on the day...so we are starting to look into that space where we can consider how the functionality that came with the system can be used to move forward" - Manager, Student Systems and Processes

- Third, it can be observed from the case of this university's SaaS CRM implementation that exploration requires proactiveness. The manager of student services reflected this:

"We are now getting proactive with how we can use the system better and other functionalities we can use, instead being reactive...there is still lots of potential in the system, it's a large system and we're only using a small part of it...but we are moving from a reactive position where we are simply responding to problems...that's not right, we've got to change things slightly to ..Ok how do we use more of the functionality that the system delivers to further improve and make changes? - Manager, Student Systems and Processes

\subsection{Reflection}

We began this study considering how organisational learning occurs as an organisation, through the use of CRM, transforms data to information and information to knowledge. We find that the exploitation/exploration attributes of such data transformation activities can influence the outcomes of the usage activities. The data indicated that use cases indicative of knowledge transfer across old user groups might be perceived as exploitation and may not generate new insights into knowledge as compared to use cases involving knowledge transfer across new and un-explored user groups. We conceived Exploitation CRM use scenario as primarily involving the improvement and refinement of current skills and capabilities within the organisation. The exploitation of CRM can therefore be concluded to be the strengthening of a firm's current customer management routines and capabilities. Exploration CRM use scenarios, on the other hand, are defined as primarily involving the generation of new customer management routines and capabilities. These may involve transformation of existing strategies, and typically challenge previous approaches. The idea of classifying CRM use scenarios based on exploitation and exploration is suggestive of ambidexterity, the expectation that organisations will adopt both learning strategies in their use of SaaS CRM. Both exploitation and exploration are relevant; the former for the codification of technological knowledge into the organisational routines and helpful for evolutionary change and incremental innovations, the latter necessary for the development of radically new technological capabilities and supporting experimentation (Khanagha et al., 2013). However, as organisations have been observed to shift between exploitation and exploration (Greve, 2007), either exploitation or exploration is expected to be dominant for a particular use scenario. Yet, due to intricacies involved in the transformation of data into useful knowledge using CRM, our findings indicate that a balance of exploitation and exploration use scenarios is necessary, if successful CRM utilisation for transforming data into useful knowledge, for enhancing customer satisfaction, is to be achieved.

The summary of our findings is that although it is expected that that organisations will adopt both exploitation and exploration organisational learning strategies in their use of SaaS CRM, only exploitation has been employed so far at Alpha University. As such the outcomes are not yet satisfactory. We find that the use of SAAS CRM at the university indicate a doing of the old with the new, primarily involving the improvement and refinement of current skills and capabilities within the organisation. This was a good outcome, and strengthened the university's current customer management routines and capabilities, more was expected from the investment in SaaS CRM in the form of new customer management routines and capabilities. For instance, transformation of existing strategies, were expected. Nevertheless, the study revealed that there is still some scope for exploration. As organisations have been observed to shift between exploitation and exploration (Greve, 2007), with either exploitation or exploration being dominant for a particular use scenario, further exploration can be expected in the future. Tthis is usually the case within organisations, desiring exploration but undertaking exploitation, this study emphasises that effective use of SaaS CRM utilisation for transforming data into useful knowledge and for enhancing customer satisfaction requires a balance of exploitation and exploration. 
The paper proposes a framework suggesting that a continuous cycle of exploration-exploitationexploration is necessary for effective utilisation of SaaS CRM. This is presented in Figure 1.

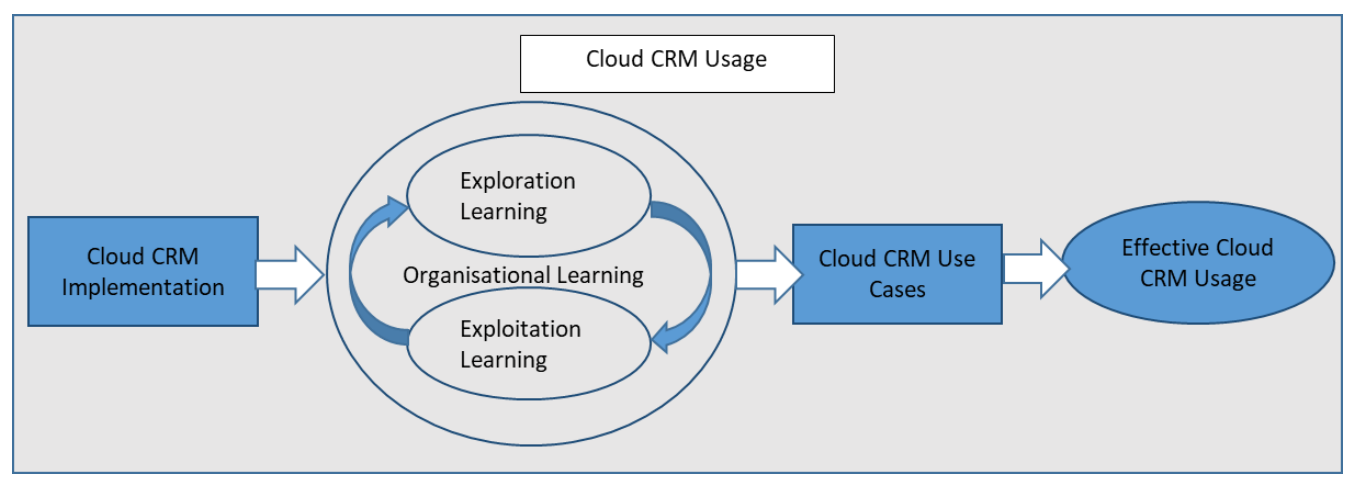

Figure 1: Framework for Organisational Learning in SaaS CRM adoption.

The framework indicates that Exploitation and Exploration learning are both significant and prerequisite to achieving benefits from SaaS CRM. Specifically, the framework emphasises that an organisation's exploitation/exploration initiates use cases that promote effective usage of SaaS CRM. This comprises a desire maximise current customer processes and knowledge via exploitation, as well as innovative and new customer processes and knowledge via exploration.

\subsection{Conclusion}

The relevance of organisational learning in CRM is an under-explored topic. Yet, CRM success relies heavily on having an adaptive organisational learning process upon which proactive decisions can be made. With arguments that organisational learning results as organisations use their SaaS CRM systems, this study purposed to consider the role of organisational learning in successful postimplementation use of SaaS CRM, with a Higher Education institution as the context of investigation. To examine how organisation learn to use SaaS CRM, we conducted a case study to investigate the idea that SaaS CRM usage may reflect organisational learning, and in turn SaaS CRM outcomes.

From the Exploitation-Exploration organisational learning perspective, the case revealed that SaaS CRM use does reflect organisational learning and informed the development of a theoretical framework for effective SaaS CRM usage. This organisational learning informed framework is a key contribution to the CRM literature, and identifies SaaS CRM implementation as the context of investigation; Organisational Learning in terms of exploitation and exploration as the constructs upon which CRM use cases are built; CRM Use Cases as the objects of investigation; and Effectiveness of SaaS CRM Use as outcomes. We observe, from the case study, that while the project's objectives show the organisation's desire to undertake exploration using SAAS CRM, the project activities and outcomes indicate that exploitation had been undertaken. The paper therefore further considered why the desired exploration had not been pursued, and observed that that exploration requires proactiveness, the right knowledge, the right level of support, and the right level of resources. Although it is expected that that organisations will adopt both learning strategies in their use of SaaS CRM, only exploitation had been employed so far, and as such the outcomes are not yet satisfactory. This emphasises that both exploitation and exploration are relevant; the former for the codification of technological knowledge into the organisational routines and helpful for evolutionary change and incremental innovations, the latter necessary for the development of radically new technological capabilities and supporting experimentation (Khanagha et al., 2013).

This insight is relevant for managers who are responsible not only for implementing the system, but for day to day use of the system for business activities. Particularly because a good balance of exploitation and exploration requires conscious planning and efforts, business managers must implement strategies that emphasise both exploitation and exploration. As a contribution to practitioners, the paper proposes that a continuous cycle of exploration-exploitation-exploration is necessary. Yet the reality is that organisations explore, exploit, and then stop exploring. In fact, rather than exploring what is already being exploited, organisations sometimes prefer to explore into a totally new system as was seen in this case. We believe that CRM managers can use our organisational learning informed model as a tool for guiding CRM use cases towards desired outcomes. We however caution against generalising the findings due to the use of a single case study. Further studies are needed involving multiple case studies across various industry segments. Likewise, surveys are to be undertaken to collect data from a range of 
organisations to establish a better understanding of how SaaS CRM use reflect an organisation's learning, and in turn SaaS CRM outcomes.

\section{References}

Amuna, Y. M. A., Shobaki, M. J. A., Naser, S. S. A., \& Badwan, J. J. (2017). Understanding Critical Variables for Customer Relationship Management in Higher Education Institution from Employees Perspective. International Journal of Information Technology and Electrical Engineering, 6(1).

Bauer, M. (2012). Lessons Learned in the Development of a CRM SaaS Solution. In A. Maedche, A. Botzenhardt, \& L. Neer (Eds.), Software for People: Fundamentals, Trends and Best Practices (pp. 257-274). Berlin, Heidelberg: Springer Berlin Heidelberg.

Benbasat, I., Goldstein, D. K., \& Mead, M. (1987). The Case Research Strategy in Studies of Information Systems. MIS Quarterly, 11(3), 369-369. Retrieved from http://search.proquest.com/docview/218119421?accountid=12528

Bernsteiner, R., Kilian, D., \& Ebersberger, B. (2016). Mobile Cloud Computing for Enterprise Systems: A Conceptual Framework for Research. International Journal of Interactive Mobile Technologies (iJIM), 1O(2), 72-76.

Bharati, P., \& Chaudhury, A. (2014). Cloud Assimilation: An Organizational Learning Approach.

Bryman, A. (2012). Social research methods (4th ed.). Newyork: Oxford university press.

Buxton, A. (2015). The reasons why SaaS will remain the dominant cloud model. TechRadar.

Chadhar, M., \& Daneshgar, F. (2018). Organizational Learning and ERP Post-implementation Phase: A Situated Learning Perspective. Journal of Information Technology Theory and Application (JITTA), $19(2), 7$.

Chadhar, M., \& Daneshgar, F. (2018). IS Enactment and organisational learning: A case of an integrated ERP post-implementation in Australia. Paper presented at the 27th Australasian Conference on Information Systems, Brsiabne.

Chen, Y.-S., Wu, C., Chu, H.-H., Lin, C.-K., \& Chuang, H.-M. (2017). Analysis of performance measures in cloud-based ubiquitous SaaS CRM project systems. The Journal of Supercomputing. doi:10.1007/s11227-017-1978-x

Edmondson, A., \& Moingeon, B. (1998). From Organizational Learning to the Learning Organization. Management Learning, 29(1), 5-20. doi:10.1177/1350507698291001

El-Gazzar, R. F. (2014). A Literature Review on Cloud Computing Adoption Issues in Enterprises. In B. Bergvall-Kåreborn \& P. A. Nielsen (Eds.), Creating Value for All Through IT: IFIP WG 8.6 International Conference on Transfer and Diffusion of IT, TDIT 2014, Aalborg, Denmark, June 2-4, 2014. Proceedings (pp. 214-242). Berlin, Heidelberg: Springer Berlin Heidelberg.

Futa, G. (2004). Customer Relationship Management system models application in higher education. Annales UMCS Informatica AI, 2(1), 445-452

Greve, H. R. (2007). Exploration and exploitation in product innovation. Industrial \& Corporate Change, 16(5), 945-975. Retrieved from http://ezproxy.lib.monash.edu.au/login?url=http://search.ebscohost.com/login.aspx?direct= true\&db=bth\&AN=27642939\&site=ehost-live\&scope $=$ site

Hunter, S. D. (2003). Information Technology, Organizational Learning, and the Market Value of the Firm. Journal Of Information Technology Theory And Application, 5(1), 1-28.

Janson, M., Cecez-Kecmanovic, D., \& Zupančič, J. (2007). Prospering in a transition economy through information technology-supported organizational learning. Information Systems Journal, 17(1), 3-36.

Kelle, U. (Ed.) (2007). Computer-assisted qualitative data analysis. London: SAGE.

Khanagha, S., Volberda, H., Sidhu, J., \& Oshri, I. (2013). Management innovation and adoption of emerging technologies: The case of cloud computing. European Management Review, 1O(1), 51-67.

Kraemmerand, P., Møller, C., \& Boer, H. (2003). ERP implementation: An integrated process of radical change and continuous learning. Production Planning \& Control, 14(4), 338-348. doi:10.1080/0953728031000117959

Lavie, D., Stettner, U., \& Tushman, M. L. (2010). Exploration and Exploitation Within and Across Organizations. The Academy of Management Annals, 4(1), 109-155. doi:10.1080/19416521003691287

Li, Y., Vanhaverbeke, W., \& Schoenmakers, W. (2008). Exploration and Exploitation in Innovation: Reframing the Interpretation. Creativity and Innovation Management, 17(2), 107-126. doi:10.1111/j.1467-8691.2008.00477.x 
Linda, A., \& Manpreet, H. (2017). Organizational Learning and Management of Technology. Production and Operations Management, 26(4), 579-590. doi:doi:10.1111/poms.12667

Malladi, S., \& Krishnan, M. S. (2012). Cloud computing adoption and its implications for CIO strategic focus-an empirical analysis.

March, J. G. (1991). Exploration and exploitation in organizational learning. Organization Science, 2(1), 71-87. Retrieved from http://ezproxy.lib.monash.edu.au/login?url=http://search.ebscohost.com/login.aspx?direct= true\&db=bth\&AN $=4433770 \&$ site $=$ ehost-live\&scope $=$ site

March, J. G. (1995). The Future, Disposable Organizations and the Rigidities of Imagination. Organization, 2(3-4), 427-440. doi:10.1177/135050849523009

March, J. G., Sproull, L. S., \& Tamuz, M. (1991). Learning from samples of one or fewer. Organization Science, 2(1), 1-13. Retrieved from http://ezproxy.lib.monash.edu.au/login?url=http://search.ebscohost.com/login.aspx?direct= true\&db=bth\&AN=443375.3\&site=ehost-live\&scope $=$ site

Oseni, T., Rahim, M. M., Smith, S. P., \& Foster, S. (2012). Exploring ERP post-implementation modifications and their influence on business process outcomes: a theory driven model. Paper presented at the 24th Australasian Conference on Information Systems, Melbourne.

Oseni, T., Rahim, M. M., Smith, S. P., \& Foster, S. (2014, June 9-11). An Initial Empirical Evaluation Of The Influence Of Erp Post-Implementation Modifications On Business Process Optimisation. Paper presented at the European Conference on Information Systems (ECIS), Tel Aviv, Israel.

Peltier, J. W., Zahay, D., \& Lehmann, D. R. (2013). Organizational Learning and CRM Success: A Model for Linking Organizational Practices, Customer Data Quality, and Performance. Journal of Interactive Marketing, 27(1), 1-13. doi:https://doi.org/10.1016/j.intmar.2012.05.001

Seethamraju, R. (2015). Adoption of software as a service (SaaS) enterprise resource planning (ERP) systems in small and medium sized enterprises (SMEs). Information systems frontiers, 17(3), 475-492.

Smith, D. M., \& Anderson, E. (2017). Hype Cycle for Cloud Computing, 2017 Retrieved from

Son, I., \& Lee, D. (2011). Assessing A New IT Service Model, Cloud Computing. Paper presented at the PACIS.

Tomblin, M. S. (2010). Theory development in enterprise systems and organizational learning. Journal of Organizational Computing and Electronic Commerce, 2O(4), 398-416. doi:10.1080/10919392.2010.516647

Vugt, T. v., \& Knasys, M. (Producer). (2015). The importance of CRM systems in Higher Education. Williams, K. J. C. (2017). Hype Cycle for Education, 2017 Retrieved from

Yamin, M., \& Sinkovics, R. R. (2007). ICT and MNE reorganisation: the paradox of control. critical perspectives on international business, 3(4), 322 - 336.

Yin, R. K. (2009). Case study research. California: SAGE.

Copyright: [Oseni, Chadhar, Ivkovic, Firmin] (C) 2018 authors. This is an open-access article distributed under the terms of the Creative Commons Attribution-NonCommercial 3.0 Australia License, which permits non-commercial use, distribution, and reproduction in any medium, provided the original author and ACIS are credited. 